\title{
artigo
}

Silva, A.P.; Camacho, A.C.L.F.; Menezes, Menezes, H.F.; Santos, A.C.F.T.; Moniz, M.A.; Santos, R.D.; Panetto, O.S.;

Riscos à saúde do trabalhador rural exposto ao agrotóxico

DOI: https://doi.org/10.36489/saudecoletiva.2020v10i52p2094-2111

\section{Riscos à saúde do trabalhador rural exposto ao agrotóxico}

\author{
Health risks from rural workers exposed to pesticides \\ Riesgos de salud del trabajadores rurales expuestos a plaguicidas
}

\begin{abstract}
RESUMO
O objetivo é analisar a prevalência de fatores de risco à saúde do trabalhador rural exposto ao agrotóxico. Trata-se de estudo transversal, observacional, que ocorreu no período de março a agosto de 2019 e cuja amostra foi constituída de 60 trabalhadores rurais do município de Casimiro de Abreu, no estado do Rio de Janeiro. Identificou-se, que o trabalhador rural tinha um tempo de contato com agrotóxicos de 30,1 anos, e 54,3\% dos participantes relataram apresentar algum sintoma de intoxicação (cefaleia, dispneia, prurido pelo corpo, náusea e sinusite). 0 glifosato é o agrotóxico mais utilizado no município e a maioria dos trabalhadores entrevistados não utiliza Equipamento de Proteção Individual adequado durante a manipulação dos agroquímicos. Evidenciou-se a necessidade de mecanismos alternativos para as práticas de cultivo no agronegócio, e de cuidado com a saúde do trabalhador exposto ao uso de agrotóxicos por meio da adoção de medidas educativas e preventivas. Recomenda-se aos órgãos públicos a implantação de programas específicos para o trabalhador rural, na perspectiva única de permanecer no ranking do agronegócio, porém com práticas de promover a saúde integral desta população rural vulnerável em sua totalidade.
\end{abstract}

DESCRITORES: Agrotóxicos; Exposição Ambiental; Saúde do Trabalhador Rural.

\section{ABSTRACT}

The aim is to analyze the prevalence of risk factors for the health of rural workers exposed to pesticides. This is a cross-sectional, observational study, which took place from March to August 2019 and whose sample consisted of 60 rural workers from the municipality of Casimiro de Abreu, in the state of Rio de Janeiro. It was identified that the rural worker had 30.1 years of contact with pesticides, and $54.3 \%$ of the participants reported having some intoxication symptoms (headache, dyspnea, itchy body, nausea and sinusitis). Glyphosate is the most widely used pesticide in the municipality and most of the workers interviewed do not use adequate Personal Protective Equipment when handling agrochemicals. The need for alternative mechanisms for cultivation practices in agribusiness was highlighted, as well as care for the health of workers exposed to the use of pesticides through the adoption of educational and preventive measures. Public agencies are recommended to implement specific programs for rural workers, with the unique perspective of remaining in the agribusiness ranking, but with practices to promote the integral health of this vulnerable rural population in its entirety.

DESCRIPTORS: Pesticides; Environmental Exhibition; Rural Worker's Health.

\section{RESUMEN}

El objetivo es analizar la prevalencia de factores de riesgo para la salud de los trabajadores rurales expuestos a pesticidas. Este es un estudio transversal, observacional, que tuvo lugar de marzo a agosto de 2019 y cuya muestra consistió en 60 trabajadores rurales del municipio de Casimiro de Abreu, en el estado de Rio de Janeiro. Se identificó que el trabajador rural tuvo 30.1 años de contacto con pesticidas, y el $54.3 \%$ de los participantes informaron tener algunos síntomas de intoxicación (dolor de cabeza, disnea, picazón en el cuerpo, náuseas y sinusitis). El glifosato es el pesticida más utilizado en el municipio y la mayoría de los trabajadores entrevistados no utilizan equipos de protección personal adecuados cuando manipulan agroquímicos. Se destacó la necesidad de mecanismos alternativos para las prácticas de cultivo en los agronegocios, así como el cuidado de la salud de los trabajadores expuestos al uso de pesticidas mediante la adopción de medidas educativas y preventivas. Se recomienda que las agencias públicas implementen programas específicos para los trabajadores rurales, con la perspectiva única de permanecer en el ranking de agronegocios, pero con prácticas para promover la salud integral de esta población rural vulnerable en su conjunto.

DESCRIPTORES: Pesticidas; Exposición Ambiental; Salud del Trabajador Rural.

RECEBIDO EM: 04/11/2019 APROVADO EM: 05/11/2019

\section{Angélica Pinto da Silva}

Farmacêutica Sanitarista. Mestranda. Programa Acadêmico em Ciências do Cuidado em Saúde. Escola de Enfermagem Aurora de Afonso Costa. Universidade Federal Fluminense - UFF/RJ. https://orcid.org/0000-0002-1872-2162 


\section{Alessandra Conceição Leite Funchal Camacho}

Enfermeira. Doutora. Docente no Programa Acadêmico em Ciências do Cuidado em Saúde. Escola de Enfermagem Aurora de Afonso Costa. Universidade Federal Fluminense - UFF/RJ. https://orcid.org/0000-0001-6600-6630

\section{Harlon França de Menezes}

Enfermeiro. Doutorando. Programa Acadêmico em Ciências do Cuidado em Saúde. Escola de Enfermagem Aurora de Afonso Costa. Universidade Federal Fluminense - UFF/RJ. https://orcid.org/0000-0001-9884-6511

\section{Ana Cláudia Felipe Thomaz dos Santos}

Enfermeira. Mestranda. Programa Acadêmico em Ciências do Cuidado em Saúde. Escola de Enfermagem Aurora de Afonso Costa. Universidade Federal Fluminense - UFF/RJ. https://orcid.org/0000-0001-8960-4545

\section{Marcela de Abreu Moniz}

Enfermeira. Doutora. Docente na Residência em Enfermagem em Saúde Coletiva na área de Ambiente, Saúde e Trabalho. Escola de Enfermagem Aurora de Afonso Costa. Universidade Federal Fluminense - UFF/RJ. https://orcid.org/0000-0002-8481-7258

\section{Rene Dias dos Santos}

Farmacêutico. Especialista em Farmácia Clínica. Coordenador da Assistência Farmacêutica Municipal. Casimiro de Abreu/RJ. https://orcid.org/0000-0002-3645-9062

\section{Ottassano de Souza Panetto}

Biólogo. Mestre em Ciências. Fiscal do Conselho Regional de Engenharia e Agronomia. Rio de Janeiro - RJ. https://orcid.org/00000002-4524-9454

\section{INTRODUÇÃO}

0 notório avanço do agronegócio, inevitavelmente, provocou modificações profundas no processo produtivo do meio rural, as quais visam atender à competitividade econômica nas últimas décadas. Tais mudanças envolvem a mecanização das lavouras e a implantação de técnicas associadas ao aumento da utilização de agentes químicos, ou seja, dos defensivos utilizados no controle de pragas, conhecidos na literatura como agrotóxicos $^{(1)}$.

O processo de globalização acelerou as relações comerciais, e o agronegócio brasileiro ganhou destaque nas progressivas transformações econômicas pelo dinamismo na exportação, tornando-se indispensáveis ao projeto econômico de crescimento brasileiro $^{(2)}$. Esse processo trouxe uma significante modificação do ponto de vista da vida sociocultural, modificando a cultura tradicional como a urbanização e internacionalização das sociedades modernas, inclusive dos trabalhadores rurais, ranqueando o Brasil no maior consumidor de agrotóxico no mundo. De tal modo, os trabalhadores da agropecuária estão sujeitos ao monitoramento e vigilân- cia da saúde, uma vez as intoxicaçóes pelo uso de agrotóxicos são consideradas como um problema de saúde pública ${ }^{(3)}$.

De acordo com a Lei n. ${ }^{\circ} 7.802$, de 11 de julho de 1989, ainda vigente, os agrotóxicos são produtos e agentes de processos físicos, químicos ou biológicos, destinados ao uso no cultivo, armazenamento e beneficiamento de produtos agrícolas, para alterar a composição da flora ou da fauna, a fim de preservá-las da ação de seres vivos nocivos $^{(4)}$. Utilizados em larga escala na agricultura, os agrotóxicos são substâncias, às quais os trabalhadores rurais, por meio do seu manuseio, estão, potencialmente, expostos em sua rotina de trabalho ${ }^{(5)}$.

Observa-se, frequentemente, sintomas clínicos ocasionados por intoxicações agudas pelo uso de agrotóxicos, tais como: cefaleia, irritabilidade nos olhos e pele, coceira, náuseas, dores no peito, taquicardia, vertigem, tontura e dificuldade respiratória. Há refutações sobre a relação do câncer na população rural exposta aos inúmeros fatores de risco ambientais e ocupacionais ao uso de agrotóxicos. Em sua maioria, a população agrícola relata sentir os sintomas clínicos toxicológicos aos agentes químicos, porém a medicina ainda estuda e avalia os impactos a longo prazo nos organismos dos seres humanos ${ }^{(6)}$.

Os efeitos crônicos relacionados aos fatores de risco à saúde da população rural que manuseia agentes químicos são: câncer, depressão, esquecimento, problemas respiratórios graves, transtornos do sono, mal funcionamento dos rins e fígado, desequilíbrio hormonais da tireoide, impotência sexual, comprometimento fetal, do intelecto e físico ${ }^{(7,8)}$.

As intoxicações por agrotóxicos, que se verificam no local de prestação laboral, durante o trajeto ou em deslocamentos relacionados ao serviço, são classificadas como acidentes de trabalho. Correspondem aos envenenamentos intencionais ou não intencionais, decorrentes da ingestão, da inalação ou da absorção dérmica dessas substâncias químicas ${ }^{(9)}$.

Observa-se grandes produtores agrícolas se situarem no entorno das comunidades de pequenos agricultores que recebem os agrotóxicos, por contiguidade, em suas casas e nos locais de produção do seguinte modo: pela água dos canais de irrigação, que é a mesma oferecida pelo município aos moradores; pelo ar, quando vem o tempo da pulverização aérea; pelos alimentos contaminados; e pelas roupas dos trabalhadores que, a despeito da proibição 


\section{artigo}

Silva, A.P.; Camacho, A.C.L.F.; Menezes, Menezes, H.F.; Santos, A.C.F.T.; Moniz, M.A.; Santos, R.D.; Panetto, O.S.;

legal, são lavadas em casa, o que amplia a domiciliação de riscos $^{(10)}$.

Por essa razão, torna-se relevante o conhecimento a respeito da temática, com o propósito de criar subsídios voltados à educação, à assistência e à pesquisa para os profissionais de saúde, além do estímulo das perspectivas críticas, capazes de atuar nos cuidados em saúde da comunidade rural, e da contribuição com planejamento e estratégias profissionais de prevenção e promoção dessa comunidade.

Como questão norteadora, optou-se pela seguinte: Quais os fatores de risco à saúde do trabalhador rural advindos do uso de agrotóxicos? O objetivo desse estudo é analisar a prevalência de fatores de risco à saúde do trabalhador rural exposto ao agrotóxico.

\section{METODOLOGIA}

Trata-se de estudo descritivo, observacional, transversal, onde os participantes do estudo foram trabalhadores rurais residentes, e atuantes na produção agrícola, do município de Casimiro de Abreu, localizado na região da baixada litorânea, do Estado do Rio de Janeiro. O município da pesquisa possui uma população rural de 6.826 moradores atuantes na produção agrícola $^{(11)}$, e a partir de um cálculo amostral, foram selecionados 60 trabalhadores rurais participantes residentes em 03 áreas agrícolas municipais, escolhidas previamente pela Coordenação da Vigilância da Saúde do Trabalhador (VISAT): Varjão, Serra e Ribeirão.

Foram estabelecidos como critérios de inclusão: trabalhadores rurais com idade maior de 18 anos, ser trabalhador rural num período superior a um ano em território agrícola e residir no município cenário de estudo. Os critérios de exclusão foram possuir fala e/ou audição prejudicados.

Para coleta de dados, utilizou-se um questionário semiestruturado, elaborado pela pesquisadora principal, onde o participante do estudo respondeu as questões formuladas previamente e, quando necessário, o entrevistador leu em voz alta as perguntas, sem intervir nas respostas. $\mathrm{O}$

estudo permitiu obter dados elaborados e adaptados pela pesquisadora principal, com base em estudos pregressos, e teve aprovação do Comitê de Ética em Pesquisa (CEP) do Hospital Universitário Antônio Pedro (HUAP) sob parecer n. ${ }^{\circ}$ 3.036.922. O CEP encaminhou o projeto aprovado ao Comitê Nacional de Ética em Pesqui-

\section{Trata-se de}

estudo descritivo,

observacional,

\section{transversal, onde os}

participantes do estudo

foram trabalhadores

rurais residentes, $\mathrm{e}$

atuantes na produção

agrícola, do município

de Casimiro de Abreu,

localizado na região da

baixada litorânea,

do Estado do

Rio de Janeiro.

sa (CONEP) para conclusão do processo de aprovação devido à complexidade dos agentes químicos utilizados e pela relevância da saúde pública do trabalhador rural, tendo sua aprovação descrita no parecer n. ${ }^{\circ} 3.445 .242 / 2019$.

Conforme solicitação do CONEP, houve uma adequação do instrumento de coleta de dados para uma abordagem simples e clara ao entrevistado composto pelas seguintes variáveis: perfil do trabalho agrícola e dos fatores de risco: tipos de agrotóxicos, tipo de Equipamento de Proteção Individual (EPI), observação de alguns efeitos clínicos toxicológicos causados pelos agrotóxicos. Foram identificadas e analisadas as seguintes variáveis para este estudo: perfil do trabalho rural e os fatores de risco: tipos de agrotóxicos, tipo de EPI e efeitos clínicos toxicológicos causados pelos agrotóxicos.

Essa etapa foi desenvolvida durante as visitas domiciliares, com participação de duas agentes comunitárias de saúde coletando dados durante visitas as propriedades rurais dos agricultores.

No início da pesquisa, o pesquisador informou ao trabalhador rural sobre a pesquisa, seus objetivos, aspectos éticos, riscos e benefícios. Nesse momento, o entrevistado teve ciência de todos os aspectos relevantes agregados ao desenvolvimento da investigação científica, apresentando desejo ou não em participar da mesma. Não houve recusa de participação na pesquisa, o que deixou de gerar prejuízo e constrangimentos pela inviabilidade de se aplicar o instrumento relacionado à coleta de dados proposto nesse estudo.

A análise descritiva foi baseada na construção de tabelas e figuras das distribuições de frequências e cálculo de estatísticas descritivas (proporções de interesse para todas as variáveis e cálculo de mínimo, máximo, média, mediana, desvio padrão, coeficiente de variação - CV) para as variáveis quantitativas. A variabilidade da distribuição de uma variável quantitativa foi considerada baixa se $\mathrm{CV}<0,20$, moderada se $0,20 \leq C V<0,40$, e alta se $C V \geq 0,40$.

Para verificar a associação entre um fator qualitativo e a ocorrência de sintomas após uso de agrotóxicos ou fertilizantes foi usado o Teste qui-quadrado ou, quando este se mostrar inconclusivo, o Teste Exato de Fisher. $\mathrm{Na}$ análise inferencial de variáveis quantitativas, a comparação de dois grupos independentes foi feita pelo teste não paramétrico de Mann-Whitney, devido ao pequeno tamanho amostra dos grupos comparados. 
Figura 1. Produtos químicos que o trabalhador rural declarou ter contato na atividade agrícola. Casimiro de Abreu, RJ, Brasil, 2019.

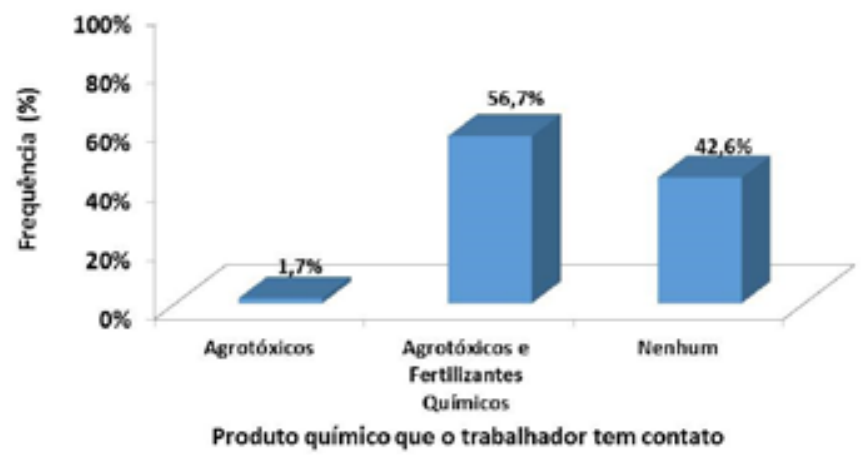

Tabela 1. Distribuição de Frequência de aspectos relacionados à saúde dos trabalhadores rurais participantes da pesquisa. Casimiro de Abreu, RJ, Brasil, 2019.

VARIÁVEL

\section{Aquisição}

Lojas de produtos agrícolas

Compra de outros agricultores

Armazenamento

Galpão

Varanda

\section{Agrotóxicos e Fertilizantes usados}

Roundup

2 Por 10

Potássio

Outros

Idade que começou a ter contato com agrotóxicos

$$
\begin{aligned}
& 10 \mid-15 \\
& 15 \mid-20 \\
& 20 \mid-25 \\
& 25 \mid-30 \\
& 30 \mid-35 \\
& 35 \mid-40
\end{aligned}
$$

\section{Tempo de contato com os agrotóxicos}

이- 15

$15 \mid-30$

$30 \mid-45$

$45 \mid-60$

\section{Como prepara os agrotóxicos}

Mão sem luva

\section{RESULTADOS}

Dos 60 trabalhadores rurais entrevistados, apenas um $(1,7 \%)$ declarou que tinha contato só com agrotóxico, 34 (56,7\%) tinham contato com ambos agrotóxicos e, 25 (42,6\%) não tinham contato nem com agrotóxicos nem com fertilizantes químicos, como mostra a seguir.

A Tabela 1 traz a caracterização do contato do trabalhador rural com os agrotóxicos ou agentes químicos. A aquisição destes produtos é tipicamente feita em lojas de produtos agrícolas (90,9\%); o armazenamento é feito em galpões (97,1\%), sendo o Roundup o produto mais usado $(97,1 \%$ dos que declaram usar produtos químicos). A idade típica de início do contato com agrotóxicos é de 15 a 20 anos $(37,1 \%)$ e é mais frequente que os trabalhadores tenham contato com agentes químicos há um tempo de 30 a 45 anos $(37,1 \%)$. Os trabalhadores preparam os agrotóxicos com as mãos usando luva $(45,7 \%) \mathrm{e}$, frequentemente, usam EPI (88,6\%), destacando-se o uso de pulverizador de costas manual (65,7\%) e Botas (54,3\%).

Quanto aos efeitos clínicos, um grupo de 19 trabalhadores declarou sentir algum sintoma após o trabalho com agrotóxicos, o que resulta numa prevalência de 54,3\% entre os trabalhadores que trabalham com agentes químicos. A prevalência de cada sintoma é descrita na Figura 2. Os sintomas mais relevantes são dor de cabeça, falta de ar, coceira no corpo, enjoo e sinusite que acometem mais de $10 \%$ dos trabalhadores que tem contato com agrotóxicos em seu ambiente ocupacional.

Foi investigado se existe algum fator de risco associado à prevalência de sintomas após o uso dos agrotóxicos. A Tabela 2 mostra a prevalência de sintomas nos grupos formados de acordo com a presença e ausência de fatores que tiveram distribuição de frequências relevantes desta pesquisa. A ocorrência de sintomas nos trabalhadores rurais após o uso de agentes químicos não está significativamente associada ao sexo masculino ( $\mathrm{p}$-valor=0,347 do teste exato de Fisher), nem ao fato do agricultor saber ler ( $\mathrm{p}$-valor $=0,677 \mathrm{do}$ teste exato de Fisher), nem ao fato do produtor rural ser consumidor de bebida alcoólica ( $\mathrm{p}$-valor $=0,087$ do teste exato de Fisher), ou fumante ( $\mathrm{p}=$ valor $=0,379$ do teste exato de Fisher).

Também não são significativamente distintas as prevalências de sintomas nos grupos de tra- 


\section{artigo}

Silva, A.P.; Camacho, A.C.L.F.; Menezes, Menezes, H.F.; Santos, A.C.F..; Moniz, M.A.; Santos, R.D.; Panetto, O.S.; Riscos à saúde do trabalhador rural exposto ao agrotóxico

$\begin{array}{lcc}\text { Mão sem luva } & 9 & 25,7 \\ \text { Mão com luva } & 16 & 45,7 \\ \text { Pás } & 3 & 8,6 \\ \text { Baldes } & 7 & 20,0 \\ \text { Usa pelo menos um EPI } & 31 & 88,6 \\ \text { Pulverizador manual colocação nas costas } & 23 & 65,7 \\ \text { Botas } & 19 & 54,3 \\ \text { Calça impermeável } & 9 & 25,7 \\ \text { Máscara } & 8 & 22,9 \\ \text { Blusa impermeável } & 7 & 20,0 \\ \text { Óculos de proteção } & 5 & 14,3 \\ \text { Luvas de proteção } & 5 & 14,3 \\ \text { Pulverizador estacionário com motor } & 2 & 5,7 \\ \text { Trator pulverizador } & 1 & 2,9\end{array}$

Figura 2. Prevalências de sintomas declarados pelos agricultores ocorridos após contato com agrotóxico. Casimiro de Abreu, RJ, Brasil, 2019.

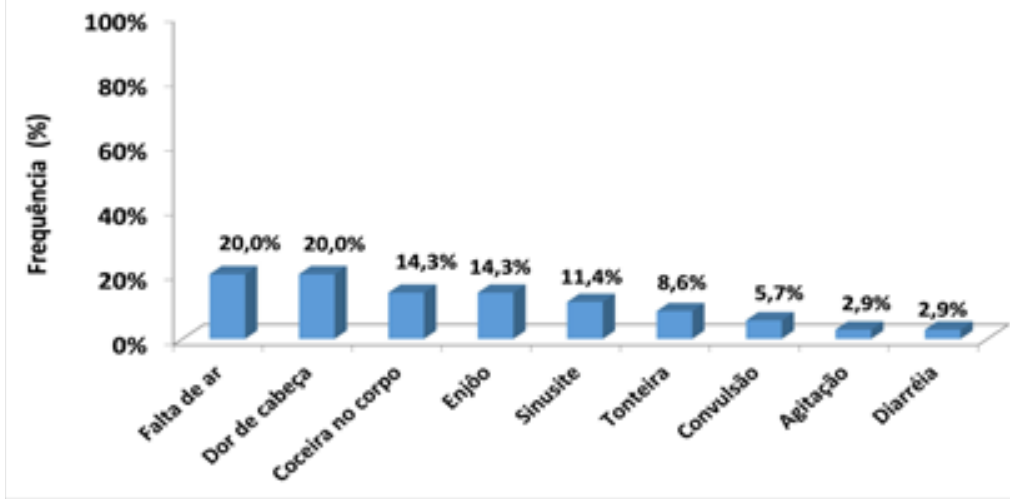

balhadores rurais que usam e de trabalhadores que não usam os equipamentos pulverizador de costas ( $\mathrm{p}$-valor $=0,311)$, botas $(\mathrm{p}$-valor $=1,000)$, luvas ( $\mathrm{p}$-valor $=0,460)$, calça impermeável ( $\mathrm{p}$-valor $=0,700)$ e máscaras ( $\mathrm{p}$-valor $=1,000)$. Os grupos determinados pelo uso de botas, luvas, calça impermeável e máscaras durante o manuseio dos agrotóxicos apresentam menores prevalências de sintomas do que os grupos de produtores rurais respectivamente complementares que não usaram tais produtos, entretanto tais diferenças não são significativas sob o ponto de vista estatístico.

A única associação significativa encontrada nesta investigação com os fatores foi a da prevalência de sintomas com o não uso da blusa impermeável: a prevalência de sintomas no grupo que usou a blusa impermeável foi de $14,3 \%$. No grupo que não usou a blusa impermeável, a prevalência de sintomas foi significativamente maior, $64,3 \%$. A diferença entre estas proporções é significativa sob o ponto de vista estatístico ( $\mathrm{p}$-valor $=0,032$ do teste Exato de Fisher). A razão de chances é igual a 0,09 e é significativa já que seu intervalo de confiança $(0,01-0,882)$ não possui o valor 1 . Logo, o uso de blusa impermeável durante o uso de agrotóxicos é um fator de proteção significativo à prevalência de sintomas após manuseio dos agentes químicos: a chance de um trabalhador rural que usa a blusa impermeável apresentar algum sintoma após o uso de agrotóxicos é 0,09 vezes a chance de um produtor rural que não usa a blusa impermeável apresentar algum sintoma após o uso de agrotóxicos em suas lavouras.

Tabela 2. Análise da associação entre fatores qualitativos e a prevalência de algum sintoma após o uso dos agrotóxicos. Casimiro de Abreu, RJ, Brasil, 2019.

\begin{tabular}{|c|c|c|c|c|c|c|c|c|c|}
\hline \multirow[b]{2}{*}{ FATOR } & \multicolumn{3}{|c|}{ GRUPO COM AUSÊNCIA DO FATOR } & \multicolumn{3}{|c|}{ GRUPO COM PRESENÇA DO FATOR } & \multirow{2}{*}{$\begin{array}{l}\text { P-VALOR DO } \\
\text { TESTE EXATO } \\
\text { DE FISHER } \\
\text { COMPARANDO } \\
\text { AS } \\
\text { PREVALÊNCIAS } \\
\text { NOS DOIS } \\
\text { GRUPOS }\end{array}$} & \multirow[b]{2}{*}{ OR } & INTERVALO \\
\hline & $\begin{array}{l}\text { TAMANHO } \\
\text { DO GRUPO }\end{array}$ & $\begin{array}{l}\text { NÚMERO DE } \\
\text { CASOS COM } \\
\text { SINTOMAS } \\
\text { NO GRUPO }\end{array}$ & $\begin{array}{l}\text { PREVALÊNCIA } \\
\text { DE SINTOMAS }\end{array}$ & $\begin{array}{l}\text { TAMANHO } \\
\text { DO GRUPO }\end{array}$ & $\begin{array}{l}\text { NÚMERO DE } \\
\text { CASOS COM } \\
\text { SINTOMAS } \\
\text { NO GRUPO }\end{array}$ & $\begin{array}{l}\text { PREVALÊNCIA } \\
\text { DE SINTOMAS }\end{array}$ & & & $\begin{array}{l}\text { DA OR AO } \\
\text { NIIVEL DE } \\
\text { 95\% DE } \\
\text { CONFIANÇA }\end{array}$ \\
\hline $\begin{array}{l}\text { Sexo Mas- } \\
\text { culino }\end{array}$ & 5 & 4 & $80,0 \%$ & 30 & 15 & $50,0 \%$ & 0,347 & 0,25 & $0,03-2,5$ \\
\hline Sabe ler & 7 & 3 & $42,9 \%$ & 28 & 15 & $57,1 \%$ & 0,677 & 1,78 & $0,33-9,48$ \\
\hline $\begin{array}{l}\text { Consome } \\
\text { bebida } \\
\text { alcoólica }\end{array}$ & 15 & 11 & $73,3 \%$ & 20 & 8 & $40,0 \%$ & 0,087 & 0,24 & $0,06-1,04$ \\
\hline
\end{tabular}




$\begin{array}{lccccccccc}\begin{array}{l}\text { Fuma } \\ \text { Uso de Pul- }\end{array} & 29 & 17 & 58,6 \% & 6 & 2 & 33,3 \% & 0,379 & 0,35 & 0,06-2,25 \\ \begin{array}{l}\text { verizador } \\ \text { de costas }\end{array} & 12 & 5 & 41,7 \% & 23 & 14 & 60,9 \% & 0,311 & 2,17 & 0,53-9,02 \\ \begin{array}{l}\text { Uso de } \\ \text { Botas }\end{array} & 16 & 9 & 56,2 \% & 19 & 10 & 52,6 \% & 1,000 & 0,86 & 0,023-3,29 \\ \begin{array}{l}\text { Uso de } \\ \text { Luvas }\end{array} & 9 & 6 & 66,7 \% & 26 & 13 & 50,0 \% & 0,460 & 0,50 & 0,10-2,44 \\ \begin{array}{l}\text { Uso de } \\ \text { blusa im- }\end{array} & 28 & 18 & 64,3 \% & 7 & 1 & 14,3 \% & 0,032 & 0,09 & 0,01-0,882 \\ \begin{array}{l}\text { permeável } \\ \text { Uso de }\end{array} & 26 & 15 & 57,7 \% & 9 & 4 & 44,4 \% & 0,700 & 0,59 & 0,12-2,7 \\ \begin{array}{l}\text { Calça im- } \\ \text { permeável }\end{array} & 27 & 15 & 55,6 \% & 8 & 4 & 50,0 \% & 1,000 & 0,80 & 0,17-3,89 \\ \begin{array}{l}\text { Uso de } \\ \text { máscaras }\end{array} & 27 & & & & & & & & \end{array}$

Os resultados mostram que a presença de sintomas após o uso dos agrotóxicos está significativamente associada à idade do trabalhador ( $p$-valor $=0,034$ do teste de Man$\mathrm{n}$-Whitney). Comparando as estatísticas dos dois grupos, conclui-se que os trabalhadores que apresentam sintomas após o uso de agentes químicos são significativamente mais novos do que os trabalhadores que não apresentam sintomas após o seu uso, a diferença das médias de idade dos dois grupos é de 11 anos.

A presença de sintomas após o uso de agrotóxicos também está significativamente associada ao tempo de exposição do trabalhador a estes produtos químicos em seu ambiente ocupacional ( $\mathrm{p}$-valor $=0,031 \mathrm{do}$ teste de Mann-Whitney). Comparando as estatísticas dos dois grupos, observa-se que agricultores que apresentam sintomas após o uso de agrotóxicos apresentam tempo de exposição aos produtos significativamente menor do que o tempo de experiência dos trabalhadores que não apresentam sintomas após o uso dos agentes químicos em suas lavouras, a diferença das médias do tempo de exposição dos dois grupos é de 10,7 anos. Não há associação significativa entre a apresentação de sintomas e a idade que o trabalhador rural começou a trabalhar ( $\mathrm{p}$-valor=0,257 do teste de Mann -Whitney) e nem entre a apresentação de sintomas e a idade que o agricultor começou a trabalhar com agrotóxicos (p-va- lor=0,461 do teste de Mann -Whitney) .

Verificou-se diferença significativa de algum sintoma entre os grupos de trabalhadores que utilizaram a blusa impermeável $(64,5 \%)$ dos que não utilizaram tal vestimenta ( $\mathrm{p}$-valor $=0,03$ ).

Evidencia-se que o uso de blusa impermeável durante o uso de agrotóxicos ou fertilizantes químicos é um fator de proteção à saúde do trabalhador rural. A chance de um trabalhador que usa a blusa impermeável apresentar algum sintoma após o uso de agrotóxicos é 0,09 vezes maior que a chance de um trabalhador que não usa a blusa impermeável apresentar algum sintoma clínico.

Uma associação significativa encontrada nesta investigação com os fatores de riscos apresentados foi da ocorrência de sintomas clínicos toxicológicos, descritos pelo entrevistado, após uso de agrotóxico com o não uso da blusa impermeável: a prevalência de sintomas clínicos no grupo que usou a blusa impermeável foi de $14,3 \%$. No grupo que não usou a blusa impermeável, os sintomas clínicos toxicológicos narrados foram significativamente maiores, $64,3 \%$.

A diferença entre estas proporções é significativa sob o ponto de vista estatístico ( $\mathrm{p}$-valor $=0,032$ do teste Exato de Fisher). A razão de chances é igual a 0,09 e é significativa já que seu intervalo de confiança $(0,01-0,882)$ não possui o valor 1 . Logo, o uso de blusa impermeável durante o uso de agrotóxicos ou fertilizantes químicos é um fator de proteção significativo à prevalência de sintomas clínicos toxicológicos causados pelos agrotóxicos. A chance de um trabalhador que usa a blusa impermeável apresentar algum sintoma após o uso de agrotóxicos é 0,09 vezes a chance de um trabalhador que não usa a blusa impermeável apresentar algum sintoma clínico.

Os resultados mostram que a presença de sintomas clínicos toxicológicos após uso de agrotóxicos está significativamente associa$\mathrm{da}$ à idade do trabalhador ( $\mathrm{p}$-valor $=0,034$ ). Comparando as estatísticas dos dois grupos, conclui-se que os trabalhadores que apresentam sintomas clínicos toxicológicos causados pelos agrotóxicos são significativamente mais novos do que os trabalhadores que não apresentam sintomas, a diferença das médias de idade dos dois grupos é de 11 anos. A presença de sintomas clínicos toxicológicos causados pelos agrotóxicos também está significativamente associada ao tempo de exposição do trabalhador a estes produtos ( $\mathrm{p}$-valor $=0,031)$.

Comparando as estatísticas dos dois grupos observa-se que os trabalhadores que apresentam clínicos toxicológicos causados pelos agrotóxicos apresentam tempo de exposição a estes produtos significativamente menor do que o tempo de experiência dos trabalhadores que não apresentam sintomas, a diferença das médias do tempo de exposição dos dois grupos é de 10,7 anos. 


\section{DISCUSSÃO}

Para garantir a "saúde das frutas" e aumentar sua produtividade, os trabalhadores do agronegócio são expostos, diariamente, a elevados volumes de múltiplos agentes tóxicos, mas não dispõem de informações acerca dos fatores de risco à saúde pelo uso dessas substâncias ${ }^{(12)}$. Analisar a prevalência de fatores de risco à saúde do trabalhador rural exposto ao agrotóxico foi o objetivo desta pesquisa, resultando nos relatos que, $56,7 \%$ tem contato com agrotóxicos e/ou fertilizantes químicos, $42,6 \%$ não tinha contato e apenas 1,7\% declarou usar agrotóxico. Esses dados revelam o desconhecimento sobre os agentes químicos aplicados, assim como, do intervalo de carência, da lei da reciclagem, do uso de EPI, e operados nos rios para higienização dos equipamentos ${ }^{(13,14)}$.

Os resultados evidenciaram que os trabalhadores rurais iniciam o contato com as lavouras entre 15 e 20 anos de idade, e sua jornada de trabalho é de 35 a 40 anos de exposição ocupacional. Há uma prevalência de tempo de exposição elevada aos diversos agentes químicos e aos fatores de risco ocupacionais, através das principais vias de absorção dessas substâncias tóxicas: respiratória, cutânea, digestiva e placentária ${ }^{(15)}$. O quadro é mais preocupante e grave quando se trata dos efeitos de intoxicação crônicos pela exposição ocupacional ou ambiental aos agrotóxicos, que são menos conhecidos e mais desafiantes no estabelecimento dos nexos e suas relações. Análise abordada aos agricultores atuantes no plantio revela que, em cada safra, há utilização em média de 12 diferentes tipos de agrotóxicos ${ }^{(16)}$.

Dos participantes da pesquisa, $45,7 \%$ frequentemente usava algum tipo de EPI no preparo dos agroquímicos, destacando-se o uso de botas em $54,3 \%$, uso de calça impermeável em 25,7\% dos trabalhadores, $22,9 \%$ usava máscaras e apenas $20 \%$ narrou usar blusa impermeável, isso nos remete a uma refinada reflexão sobre a problemática. Tendo em vista, que os problemas de saúde que afetam o sistema respiratório foram indicados e representados por alterações crônicas e agudas, tais como: falta de ar, rinite alérgica, asma e doença obstrutiva crônica, sendo este sistema o mais afetado na literatura, como fator de adoecimento, o desuso ou mau uso de EPIs ${ }^{(15)}$.

Esses colaboradores se veem obrigados a reentrar nas áreas de cultivo sem que sejam respeitados, por exemplo, o tempo pós-pulverização. Muitas vezes adentram essas áreas sem portar os Equipamentos de Proteção Individual (EPIs), minimamente necessários, sendo apontada uma rápida tarefa, e sem a liberdade de recusar o trabalho inseguro, pouco protegido pelo sindicato e acossado por relações hierárquicas autoritárias ${ }^{(16)}$.

Os pesticidas são altamente voláteis diante da inalação pelas vias respiratórias, no entanto, a academia científica observa o sistema nervoso como o mais afetado ${ }^{(17)}$. Há estudos que revelam intoxicação dérmica pelo contato direto das mãos no preparo dos agentes químicos, pelo não uso de roupas adequadas, assim como, pelos EPIs estarem contaminados por resíduos dos produtos químicos utilizados no plantio ${ }^{(18)}$.

Entre os adoecimentos narrados pelos trabalhadores, vimos: cefaleia, tonteira, convulsão, agitação, e até neuropatia periférica, visto que os compostos químicos podem entrar pela via dérmica ou pela via respiratória e realizar a inibição de acetilcolinesterase (ACE), ou seja, atingir as terminações nervosas, fator relacionado aos EPIs que intensifica a problemática. A importância de se usar EPIs, está na inibição da $\mathrm{ACE}$, posto que, existe acúmulo de neurotransmissores da acetilcolina (AC) nas sinapses, evidenciando que há prejuízos no sistema nervoso central dos seres humanos expostos ${ }^{(18,19)}$.

A intensa mobilização da sociedade tem gerado a realização de audiências públicas; de debates; de produção e difusão ampliada de informações; e da proibição de pulverização aérea em alguns municípios. Esses movimentos civis se constituem não só em ações concretas, as quais as políticas públicas de governo encontram-se incapazes de realizar, mas também são reconhecidos pelo seu importante papel na proteção da saúde pública ${ }^{(18)}$.

A aquisição destes produtos é tipicamente feita em 90,9\% em lojas de produtos agrícolas pelo trabalhador sem apresentação de receita específica para compra deste agente químico, normalmente emitido por um engenheiro agrônomo, como cita a legislação vigente. Consequentemente, as informações adequadas são omitidas pela ausência desse profissional capacitado, impactando diretamente na demonstração correta do uso, a dosagem apropriada desses produtos químicos, e o tempo de exposiçãoo ${ }^{(20,21)}$. Porém a questão financeira remete a uma limitação na aquisição dos EPIs, sabendo que a grande maioria dos agricultores apresenta renda familiar entre dois e três salários mínimos ${ }^{(22)}$.

Estudos epidemiológicos, os quais foram realizados com a exposição aos agrotóxicos e com ensaios in vitro e in vivo, demonstraram que os herbicidas e os inseticidas atuam, com uma margem que varia entre 05 e 10 casos, nos mecanismos de carcinogenicidade relevantes ${ }^{(23)}$. Esse dado é relevante para a análise da problemática em questão, uma vez que os herbicidas $^{(22)}$ : "glifosato e 2,4,D" foram os agrotóxicos citados nessa pesquisa e são usados concomitantemente com autorização legal, no meio rural e urbano, adição à formulação de produtos comerciais ${ }^{(24)}$.

De acordo com o Sistema de Informações sobre Agrotóxicos da Agência Nacional de Vigilância Sanitária, dos 10 princípios ativos mais usados, cinco são considerados altamente tóxicos (Classe Toxicológica II); um é considerado extremamente tóxico (Classe Toxicológica I); dois, medianamente tóxicos (Classe III); e dois, pouco tóxicos (Classe IV). $\mathrm{O}$ agente químico mais citado nesta pesquisa foram os herbicidas "glifosato e 2,4, Dinitrofenol", apresentado respectivamente, Classe IV e Classe II ${ }^{(25)}$.

Em curto prazo, a negação de riscos caracterizada como uma estratégia defensiva serve como uma possível "proteção" para a saúde mental desses agricultores, os quais passam a acreditar na inexistência de riscos diretos à sua saúde ${ }^{(26)}$, por mais que as evidências $\mathrm{e}$ as informações disponíveis lhes mostrem o contrário. Em médio e longo prazos, entretanto, essas estratégias defensivas acabam por levar os trabalhadores a, voluntariamente, colocarem-se em situações de risco diante dos perigos do trabalho, o que configura uma situação de difícil gerenciamento para a vigilância da saúde, no caso de não haver o pleno entendimento de como essas estra- 
tégias defensivas são formadas dentro desses grupos populacionais específicos ${ }^{(24)}$.

É importante ressaltar que esses dados apresentados não sejam somente utilizados para o planejamento de ações mitigadoras e de gerenciamento de riscos, mas também na construção de políticas educativas que priorizem uma comunicação de risco associado ao uso de agrotóxico com clareza e eficácia, direcionada à promoção da saúde dessas populações expostas no país.

Sobre análise de risco ${ }^{(27)}$, há relevância na educação permanente no conhecimento dos fatores risco, quanto mais se familiarizam com ele, habitualmente, tem-se uma tolerância maior consigo. Fato, particularmente, preocupante, pois quando se tem muita familiaridade com os riscos, geralmente, a sua percepção torna-se reduzida e, por conseguinte, diminuem-se os procedimentos de segurança e controle.

No convívio diário com situações, potencialmente, danosas à saúde e ao meio ambiente, nota-se uma atitude de tolerância por parte dos moradores rurais(28). Por sua vez, os moradores da área urbana, apesar de não terem uma relação direta com o uso de agrotóxicos, demonstram grande preocupação com as condições sanitário-ambientais, no que tange à exposição.

\section{CONCLUSÃO}

O estudo pôde avaliar os riscos de exposição à saúde do trabalhador rural mediante ao manuseio dos agrotóxicos em um município da região da baixada litorânea do Estado do Rio de Janeiro. Demonstrou-se resultados que corroboram com o perfil da exposição ocupacional dos agricultores, com ênfase ao uso de múltiplos produtos químicos de elevada toxicidade por período prolongado, trabalhador desprovido da utilização dos EPIs propostos e, desconsiderando o tempo de reentrada, principalmente, para colheita.

Atesta-se uma incidência de 54,3\% maior nos agricultores que declararam ter algum sintoma após uso de agrotóxicos, e os sintomas de intoxicação mais relevantes, dentre eles: dor de cabeça, falta de ar, coceira no corpo, enjoo e sinusite; e menor incidência de sintomas do que os grupos que não usaram tais produtos.

Observar apenas a vulnerabilidade ao uso indiscriminado dos pesticidas e a relação dos riscos à saúde do trabalho agrícola exposto ao manuseio dos agrotóxicos não pode ser minimizado apenas pela conscientização do uso adequado dos EPIs específicos na jornada de trabalho. Há urgência num olhar das ações de promoção da saúde aos trabalhadores rurais pelo governo, instituições, indústrias farmacêuticas e órgãos públicos, no que tange à prevenção de riscos ocupacionais relacionados ao uso indiscriminado dos agrotóxicos, em conformidade com a preservação do meio ambiente. As problemáticas relacionadas aos agrotóxicos deve ser prontamente reconhecida, valorizando-se o contexto de fragilidade social, de exposição ambiental e humana aos agrotóxicos.

São necessárias abordagens que conside- rem todos fatores de riscos dos danos à saúde da população em pesquisa, o que implica a implementação de políticas e ações específicas de promoção da saúde e à educação do agricultor, como por exemplo: informações sobre alimentação, momentos de lazer, prática esportivas, condições dignas de trabalho e formação de grupos sociais nas comunidades rurais.

As aprovações indiscriminadas de uso dos inúmeros agrotóxicos necessitam ser reavaliados em conjunto pelas autoridades competentes, superando os interesses pessoais, assim como o discurso hegemônico pelo desuso dos agrotóxicos. Distingue-se uma imediata e efetiva adequação das políticas públicas, no qual a sociedade tem um papel importante sobre o os aspectos legais, financeiros e do enfoque agroecológico, que valoriza o crescimento econômico, a equidade social e a proteção ambiental.

Por fim, é de grande valia para desenvolvimento social e preventivo a diversificação dos profissionais, como os do meio ambiente, da agricultura, da educação, da ação social, entre outros; devem atuar mais em parceria com a saúde, sendo fundamental planejar ações, capacitações e informações sobre os cuidados em saúde e de prevenção de agravos ligados à utilização de agrotóxicos na agricultura. Recomenda-se aos órgãos públicos a implantação de programas específicos para o trabalhador rural, na perspectiva única de permanecer no ranking do agronegócio, porém com práticas de promover a saúde integral desta população rural vulnerável em sua totalidade.

\section{REFERÊNCIAS}

1. Martinelli G et al. Agricultora no Brasil [Internet]. 2011. [cited 2018 Apr 2]. Available from: http://ipea.gov.br/agencia/images/stories/ PDFs/livros/livros/170404_livro_agricultura_no_Brasil_capitulo6.pdf.

2. Viero CM et al. Sociedade de risco: o uso dos agrotóxicos e implicações na saúde do trabalhador rural. Esc Anna Nery [Internet]. 2016 Mar [cited 2018 Apr 6]; 20(1):99-105. Available from: http:/www.scielo.br/scielo.php?script=sci_arttext\&pid=S1414-81452016000100099.

3. Stotz EN. Os limites da agricultura convencional e as razões de sua persistência: estudo do caso de Sumidouro, RJ. Rev Bras Saúde Ocup [Internet]. 2012 Jun [cited 2018 Mar 13]; 37(125): 114-26.
Available from: http:/www.scielo.br/scielo.php?script=sci_arttext\&pid=S0303-76572012000100014.

4. Brasil. Lei n. ${ }^{\circ} 7.802$, de 11 de julho de 1989 [Internet]. [cited 2018 Mar 5]. Available from: http://www.agricultura.gov.br/assuntos/insumos-agropecuarios/insumos-agricolas/agrotoxicos/legislacao/ arquivos-de-legislacao/lei-7802-1989-lei-dos-agrotoxicos.

5. Selmi GFR, Trapé AZ. Proteção da saúde de trabalhadores rurais: a necessidade de padronização das metodologias de quantificação da exposição dérmica a agrotóxicos. Cad Saúde Pública [Internet]. 2014 [cited 2018 Mar 5]; 30(5):952-60. DOI: https://doi.org/10.1590/ 0102-311X00168312.

6. Morin PV, Stumm EMF. Transtornos mentais comuns em agri- 


\section{artigo}

Silva, A.P.; Camacho, A.C.L.F.; Menezes, Menezes, H.F.; Santos, A.C.F.T.; Moniz, M.A.; Santos, R.D.; Panetto, O.S.;

Riscos à saúde do trabalhador rural exposto ao agrotóxico

\section{REFERÊNCIAS}

cultores, relação com agrotóxicos, sintomas físicos e doenças preexistentes. Revista eletrônica PSICO, PUCRS [Internet]. 2018 [cited 2018 Mar 5]; 49(2): 196-206. Available from: http://revistaseletronicas.pucrs.br/ojs/index.php/revistapsico/index.

7. Instituto Nacional de Câncer José Alencar Gomes da Silva. Coordenação de Prevenção e Vigilância Estimativa 2019. Incidência de Câncer no Brasil. Rio de Janeiro: INCA, 2019.

8. Santana VS, Moura MCP, Nogueira FF. Mortalidade por intoxicação ocupacional relacionada a agrotóxicos, 2000-2009, Brasil. Rev Saúde Pública [Internet]. 2013 Jun [cited 2018 Apr 18]; 47(3):598-606. DOI: http://dx.doi.org/10.1590/S0034-8910.2013047004306.

9. Meneguel SN, et al . Características epidemiológicas do suicídio no Rio Grande do Sul. Rev Saúde Pública [Internet]. 2014 Dec [cited 2018 Mar 27]; 38(6):804-10. Available from: http://www.scielo.br/ scielo.php?script=sci_arttext\&pid=\$0034-89102004000600008.

10. Soares W, Porto MFS. Uso de agrotóxicos e impactos econômicos sobre a saúde. Rev Saúde Pública [Internet]. 2012 [cited 2018 Mar 29]; Apr; 46(2):209-17. DOI: http://dx.doi.org/10.1590/S003489102012005000006.

11. Instituto Brasileiro de Geografia e Estatística. Pesquisa de Informações Básicas Municipais [Internet]. Rio de Janeiro: IBGE; 2010 [acesso em 02 jun. 2018]. Disponivel em: http://www.ibge.gov.br/ home/estatistica/economia/perfilmunic/default.shtm.

12. Gregolis TBL, Pinto WJ, Peres F. Percepção de riscos do uso de agrotóxicos por trabalhadores da agricultura familiar do município de Rio Branco, AC. Rev Bras Saúde Ocup [Internet]. 2012 Jun [cited 2018 Apr 25]; 37(125):99-113. Available from: http://www.scielo.br/ scielo.php?script=sci_arttext\&pid=S0303-76572012000100013.

13. Gonzaga MC, Santos SO. Avaliação das condições de trabalho inerentes ao uso de agrotóxicos nos municípios de Fátima do Sul, Glória de Dourados e Vicentina - Mato Grosso do Sul - 1990. Rev. Bras. Saúde Ocup. 1992; 20(76):42-46.

14. Rigotto RM et al. O verde da economia no campo: desafios à pesquisa e às políticas públicas para a promoção da saúde no avanço da modernização agrícola. Ciência \& Saúde Coletiva [Internet]. 2012 [cited2018Apr5]; 17(6):1533-542. Availablefrom:https:/scielosp.org/ scielo.php?script=sci_arttext\&pid=S1413-81232012000600017.

15. Hoshino ACH, Ferreira HP, Taguchi CK, Tomita S, Miranda MF. Estudo da ototoxicidade em trabalhadores expostos a organofosforados. Revista Brasileira Otorrinolaringologia [Internet]. 2015 [citado 2016 jan 15]; 74(6):912-8. Disponível em: DOI: http://dx.doi. org/10.1590/S0034-72992008000600015.

16. Faria NMX, Rosa JAR, Facchini LA. Intoxicações por agrotóxicos entre trabalhadores rurais de fruticultura, Bento Gonçalves, RS. Rev. Saúde Pública [Internet]. 2014 [cited 2018 Nov 11]; 43(2):335-344. DOI: http://dx.doi.org/10.1590/S0034-89102009005000014.

17. Siqueira DF, Moura RM, Laurentino GEC, Araújo AJ, Cruz SL. Análise da exposição de trabalhadores rurais a agrotóxicos. Rev. bras. Promoc. Saúde [Internet]. 2013 abr.-jun. [cited 2018 Mar 5]; 26(2):182-191. Available from: https://pesquisa.bvsalud.org/brasil/ resource/pt/lil-706455.
18. Meirelles LA, et al. A contaminação por agrotóxicos e o uso de epi: análise de aspectos legais e de projeto. Revisões Temáticas [Internet]. 2016 [cited 2018 Mar 5]; XII(2):75-82. DOI: http://dx.doi. org/10.15667/laborealxii0216lam.

19. Rigoto RM, et al. Uso de agrotóxicos no Brasil e problemas para a saúde pública. Ciência \& Saúde Coletiva [Internet]. 2012 [cited 2019 Jul 5]; 30(7):1-3. Available from: http://www.scielo.br/pdf/csp/ v30n7/pt_0102-311X-csp-30-7-1360.pdf.

20. Fenzke MN, Mello MCVA, Santos KNSC, et al. Adoecimentos e fatores relacionados à saúde do trabalhador rural. Revista Enfermagem UFPE on-line [Internet]. 2018 ago. [cited 2018 Nov 11]; 12(8):2214-26. DOI: https://doi.org/10.5205/1981-8963-v12i8a23 1532p2214-2226-2018.

21. Hungaro AA, Correa LM, Silvino MCS, Rocha SM, Martins BF, Oliveira MLF. Pesticide poisoning: records of a toxicological assistance sentinel service. Ciênc Cuid Saude [Internet]. 2015, July-Sept [cited 2017 Oct 20];14(3):1362-9. Available from:http://periodicos.uem.br/ ojs/index.php/CiencCuidSaude/article/view/25119/15391.

22. Santos AO, Borges-Paluch LR, Cerqueira TPS, Teles ALB. Utilização de equipamentos de proteção individual e agrotóxicos por agricultores de município do Recôncavo Baiano. Revista da Universidade Vale do Rio Verde [Internet]. 2017, Jan-July [cited 2017 Oct 20];15(1):738-54. Available from: http://periodicos.unincor.br/index. php/revist aunincor/article/view/3309/pdf_665.

23. Brust RS, Oliveira LPM, Silva ACSS, Regazzi ICR, Aguiar GS, Knupp VMAO. Epidemiological profile of farmworkers from the state of Rio de Janeiro. Rev Bras Enferm [Internet]. 2019 [cited 2019 Dec 11] ; 72(Suppl 1):122-8. DOI: http://dx.doi.org/10.1590/0034-71672017-0555.

24. Carneiro FF, et al. Mapeamento de vulnerabilidades socioambientais e de contextos de promoção da saúde ambiental na comunidade rural do Lamarão, Distrito Federal, 2011. Rev Bras Saúde Ocup [Internet]. 2012 Jun [cited 2018 Apr 2]; 37(125):143-48. Available from: http:/www.scielo.br/scielo.php?script=sci_arttext\&pid=S0303-76572012000100016.

25. Agência Nacional de Vigilância Sanitária (Anvisa). Guia para elaboração de rótulo e bula de agrotóxicos, afins e preservativos de madeira. Agrotóxicos - Guia, Brasilia: ANVISA. 2018 jan.; 1(12):10-11.

26. Silva AP, Camacho ACLF, et al. Exposição do trabalhador rural ao uso de agrotóxicos: uma revisão integrativa. Rev. Saúde Coletiva (Barueri). 2019 set; 9(49):1576-1584.

27. Siqueira DF, MR, Laurentino GEC, Araújo AJ, Cruz SL. Analise da exposição de trabalhadores rurais a agrotóxicos. Rev Bras Promoç Saude (Impr.) [Internet]. 2013 Apr-Jun [cited 2018 Mar 31]; 26(2):182-91. Available from: http://pesquisa.bvsalud.org/brasil/ resource/pt/lil-706455.

28. Bohner TOL. Agrotóxicos e sustentabilidade: percepção dos sujeitos sociais no meio rural [dissertação]. Rio Grande do Sul: Universidade Federal de Santa Maria, Centro de Ciências Rurais [Internet]; 2015 [cited 2019 Nov 11]. 103 p. Available from: http://w3.ufsm.br/ ppgexr/images/dissertacoes/Disserta\%C3\%A7\%C3\%A30_TANNY_ OLIVEIRA_LIMA_BOHNER.pdf. 